\title{
FINITE EXTENSIONS OF ABELIAN GROUPS WITH MINIMUM CONDITION
}

\author{
BY \\ REINHOLD BAER
}

Among the groups with minimum condition one meets, often quite unexpectedly, groups which satisfy one of the following two conditions:

(a) the commutator subgroup is finite;

(b) there exists an abelian subgroup of finite index.

It is our objective in this investigation to give various characterizations of these two classes of groups some of which have very little obvious connection with either the minimum condition or properties (a) and (b).

Our principal result, stated in $\S 0$, contains characterizations of the groups with minimum condition and finite commutator subgroup; and the proof of this theorem is effected in $\S 1$ to $\S 5$. In $\S 6$ we specialize this result to show that torsion groups with finite automorphism groups are finite. In $\$ 7$ we enunciate a characterization of the groups with minimum condition possessing abelian subgroups of finite index; and the proof of this proposition will be effected in $\S 8$ to $\$ 11$. These results are used in $\$ 12$ to show that the $p$-Sylow subgroups of these groups are conjugate.

0 . In this section we enunciate and discuss our principal

THEOREM. The following properties of the group $G$ are equivalent. finite.

(i) $G$ is a torsion group and every torsion group of automorphisms of $G$ is

(ii) $G / Z(G)$ is finite and the minimum condition is satisfied by the subgroups of $Z(G)$.

(iii) $[G, G]$ is finite and the minimum condition is satisfied by the subgroups of $G /[G, G]$.

(iv) Classes of conjugate elements in $G$ are finite and the minimum condition is satisfied by the abelian subgroups of $G$.

(v) If $J \neq 1$ is a homomorphic image of $G$, then the number of elements of squarefree order, not 1 , in $J$ is finite and positive.

Here, as always, we denote by $Z(G)$ the center and by $[G, G]$ the commutator subgroup of $G$.

REMARK 1. It is an immediate consequence of either condition (ii) or (iii) that the minimum condition is satisfied by the subgroups of $G$. On the other hand conditions (ii) and (iii) require only the validity of the minimum condition in a certain class of normal subgroups of $G$.

\footnotetext{
Received by the editors March 24, 1954 and, in revised form, October 24, 1954.
} 
REMARK 2. Subgroups and homomorphic images of groups with properties (ii) or (iii) clearly satisfy these properties too. Thus all the five properties of our theorem are inherited by subgroups and homomorphic images, a fact that is not obvious in the case of property (i).

REMARK 3. The equivalence of properties (ii) and (iii) shows that our class of groups is self-dual in the sense of MacLane.

REMARK 4. It is not an exceptional situation that every torsion group of automorphisms of a group $G$ is finite, though $G$ is not a torsion group. Denote, to construct some such example, by $R(p)$ the additive group of rational numbers of the form $i p^{-i}$ for integral $i$ and $j$. If $p_{1}, \cdots, p_{k}$ are finitely many distinct primes, then the direct sum $G=\sum R\left(p_{i}\right)$ is an abelian group which contains every $R\left(p_{i}\right)$ as a characteristic subgroup. This group possesses exactly $2^{k}$ automorphisms of finite order.

Remark 5. Suppose that $A$ is an abelian group which contains only a finite number of elements of squarefree order; and consider the group $G$ which is obtained by adjoining to $A$ an element $g$ subject to the relations:

$$
g^{4}=1 \text { and } g^{-1} a g=a^{-1} \quad \text { for every } a \text { in } A .
$$

One verifies that $G$ contains only a finite number of elements of squarefree order and that every element of the form $a g$ or $a g^{-1}$ for $a$ in $A$ has order 4 . This family of examples shows the impossibility of substituting for condition (v) the weaker condition that $G$ be a torsion group which contains only a finite number of elements of squarefree order.

REMARK 6. If the minimum condition is satisfied by the subgroups of a group $G$ [i.e. if every nonvacuous set of subgroups of $G$ contains a minimal one], then cyclic subgroups of $G$ cannot be infinite and so such a group is necessarily a torsion group [i.e. a group without elements of infinite order]. We note the important and useful fact that each of the conditions (ii), (iii), and (iv) implies in particular that $G$ is a torsion group.

1. Throughout this first section we assume that the group $G$ is a torsion group and that every torsion group of automorphisms of $G$ is finite. It is our aim then to derive the validity of (ii).

We note first that $G / Z(G)$ is a torsion group, since $G$ is a torsion group; and that $G / Z(G)$ is essentially the same as the group of inner automorphisms of $G$. Thus $G / Z(G)$ is isomorphic to a torsion group of automorphisms of $G$; and as such $G / Z(G)$ is finite.

Select next in every coset of $G / Z(G)$ a representative; and denote by $S$ the subgroup of $G$ generated by these finitely many representatives. Clearly $G=Z(G) S$ so that $G / Z(G) \simeq S /[S \cap Z(G)]$. Since $S$ is finitely generated and $S /[S \cap Z(G)]$ is finite, $S \cap Z(G)$ is finitely generated; see, for instance, Baer [5, $\$ 1$, Finiteness Principle]. Thus $S \cap Z(G)$ is a finitely generated abelian torsion group; and as such $S \cap Z(G)$ is finite. Hence $S /[S \cap Z(G)]$ and $S \cap Z(G)$ are both finite; and consequently $S$ itself is finite. Thus we have found a finite [normal] subgroup $S$ of $G$ such that $G=Z(G) S$. 
As an abelian torsion group $Z(G)$ is the direct product of its primary components $Z(G ; p)$. Denote by $P$ the finite set of primes which divide the order of the finite group $S$; and let

$$
A=\prod_{p \bigoplus_{P}} Z(G ; p), \quad B=S \prod_{p \in P} Z(G ; p) .
$$

It is clear that

$$
G=Z(G) S=\prod_{p} Z(G ; p) S=A B ;
$$

and since every $Z(G ; p)$ is part of the center of the torsion group $G$, one verifies easily that $A$ is the totality of elements in $G$ whose order is prime to the order of $S$ whereas $B$ is the totality of those elements in $G$ whose orders are divisible by primes in $P$ only. This implies in particular that $A \cap B=1$. Consequently $G$ is the direct product of $B$ and of the primary components $Z(G ; p)$ with $p$ prime to the order of $S$.

Now we consider the totality $\Sigma$ of automorphisms $\sigma$ of $G$ with the following two properties:

$$
b^{\sigma}=b \text { for every } b \text { in } B \text {; }
$$

if the prime $p$ is prime to the order of $S$ [does not belong to $P$ ], then $\sigma$ induces +1 or -1 in $Z(G ; p)$.

Since $G$ is the direct product of $B$ and of the $Z(G ; p)$ for $p$ not in $P, \Sigma$ is an abelian group of automorphisms of $G$ and $\Sigma^{2}=1$. As a torsion group of automorphisms of $G$ this group $\Sigma$ is finite; and consequently there exists only a finite number of primes $p$ which are prime to the order of $S$ such that $Z(G ; p) \neq 1$. Since $A$ is the totality of elements in $G$ whose order is prime to the order of $S$, and since $B$ is the totality of elements in $G$ whose orders are divisible by primes in $P$ only, we see that we have verified the following fact:

${ }^{*}$ ) There exists only a finite number of primes $p$ such that $G$ contains elements of order $p$.

Consider now some definite prime $q$. The totality of elements of order $q$ [or 1 ] in $Z(G)$ is a characteristic subgroup $Q$ of $G$. To prove the finiteness of $Q$ we distinguish two cases.

CASE 1 . There exists a normal subgroup $N$ of $G$ such that $[G: N]=q$.

We consider the totality $\theta$ of automorphisms $\sigma$ of $G$ with the following properties:

$$
\begin{aligned}
& x^{\sigma}=x \text { for every } x \text { in } N ; \\
& g^{\sigma} \equiv g \text { modulo } Q \text { for every } g \text { in } G .
\end{aligned}
$$

It is fairly obvious that $\theta$ is a group of automorphisms of $G$. Since $G / N$ is a cyclic group of order $q$, since $Q \leqq Z(G)$ and $Q^{q}=1$, it is easy to see that $Q$ and $\theta$ are isomorphic groups. Hence $\theta$ is a torsion group of automorphisms of $G$; and as such $\theta$ is finite. The isomorphic group $Q$ is therefore finite too. 
CASE 2. There does not exist a normal subgroup of $G$ whose index is $q$.

We recall the existence of a finite subgroup $S$ of $G$ such that $G=Z(G) S$; and we denote by $M$ the product of $S$ and of all the primary components $Z(G ; p)$ for $p \neq q$. Since $G=M Z(G ; q)$ and $Z(G ; q) \leqq Z(G), M$ is a normal subgroup of $G$. Consequently

$$
\begin{aligned}
G / M Z(G ; q)^{q} & =M Z(G ; q) / M Z(G ; q)^{q} \simeq Z(G ; q) /\left[Z(G ; q) \cap M Z(G ; q)^{q}\right] \\
& \simeq Z(G ; q) /[Z(G ; q) \cap M] Z(G ; q)^{q}
\end{aligned}
$$

so that $G / M Z(G ; q)^{q}$ is an abelian group whose $q$ th power is 1 . But such a group is either 1 or else it possesses a [normal] subgroup of index $q$; and now we deduce $G=M Z(G ; q)^{q}$ from the hypothesis of Case 2 . This implies in particular that $Z(G ; q) \leqq S Z(G ; q)^{q}$; and we deduce the finiteness of $Z(G ; q) / Z(G ; q)^{q}$ from the finiteness of $S$. Thus $Z(G ; q)$ is an abelian $q$-group with finite $Z(G ; q) / Z(G ; q)^{q}$; and it is well known that such a group is the direct product of finitely many cyclic groups and of groups of type $q^{\infty}$. Since $S \cap Z(G ; q)$ is finite, there exists consequently a direct decomposition $Z(G ; q)$ $=U \otimes V$ with the following properties:

$S \cap Z(G ; q) \leqq U$;

$U$ contains only a finite number of elements of order $q$;

$V=V^{q}$ is a direct product of groups $V$, of type $q^{\infty}$.

If we recall the definition of $M$, then we verify easily that $G=(M U) V$ and that

$V \cap M U=V \cap S U=V \cap Z(G ; q) \cap S U=V \cap[Z(G ; q) \cap S] U=V \cap U=1$.

Hence $G$ is the direct product of $M U$ and $V$ and consequently of $M U$ and the groups $V_{\nu}$ of type $q^{\infty}$.

Consider now the totality $\phi$ of automorphisms $\sigma$ of $G$ with the following properties:

$$
\begin{aligned}
& \sigma \text { induces }+1 \text { or }-1 \text { in } V_{\nu} \\
& x^{\sigma}=x \text { for } x \text { in } M U .
\end{aligned}
$$

One verifies easily that $\phi$ is an abelian group of automorphisms of $G$ and that $\phi^{2}=1$. Thus $\phi$ is a torsion group of automorphisms of $G$; and as such $\phi$ is finite. Since $G$ is the direct product of $M U$ and of the groups $V_{\nu}$ of type $q^{\infty}$, the finiteness of $\phi$ implies the finiteness of the number of direct factors $V_{\nu}$. Thus $V$ too contains only a finite number of elements of order $q$. Since $Z(G ; q)$ is the direct product of groups $U$ and $V$ each of which contains only a finite number of elements of order $q$, the group $Z(G ; q)$ itself contains only a finite number of elements of order $q$. Thus we have verified in either case the finiteness of $Q$.

By $\left({ }^{*}\right)$ there exists only a finite number of primes $p$ such that $Z(G)$ contains elements of order $p$; and by what we have shown just now it follows 
that $Z(G)$ contains only a finite number of elements of squarefree order. It is well known that the minimum condition is satisfied by the subgroups of the abelian group $Z(G)$ if, and only if, $Z(G)$ is a torsion group containing only a finite number of elements of squarefree order. Thus we have shown that (ii) is a consequence of (i).

REMARK. For what it is worth it may be pointed out that once we had proved the finiteness of $G / Z(G)$ we applied condition (i) only on abelian torsion groups of automorphisms of $G$.

2. The following lemma which we shall need below is not a special case of our principal theorem.

Lemma. If $P$ is an abelian $p$-group and $P=P^{p}$, if the automorphism $\sigma$ of $P$ leaves invariant every element of order $p^{2}$ in $P$, and if the order of $\sigma$ is finite, then $\sigma=1$.

Proof. If $\sigma$ were not 1 , then there would exist a positive integer $k$ such that $\sigma^{k}=\tau$ has order a prime $q$. Since every element of order $p$ in $P=P^{p}$ is the $p$ th power of an element of order $p^{2}$ in $P$,

$$
x^{p^{2}}=1 \text { implies } x^{\tau}=x .
$$

Since $\tau \neq 1$, there exists an element $w$ of minimal order $p^{m}$ which is not a fixed element of the automorphism $\tau$ of $P$. Clearly $2<m$; and every element whose order divides $p^{m-1}$ is a fixed element of $\tau$. Let $v=w^{r-1}$. Since $w^{p}$ has order $p^{m-1}$, it is a fixed element of $\tau$. Hence

$$
w^{p}=w^{p \tau}=w^{\tau p}=w^{p} v^{p} \text { or } v^{p}=1 .
$$

Consequently $v$ is a fixed element of $\tau$; and this implies

$$
w^{r^{i}}=w v^{i} \text { for every positive } j \text {. }
$$

But $q$ is the order of $\tau$. Hence

$$
w=w^{\tau^{q}}=w v^{q} \text { or } v^{q}=1 .
$$

Since $v \neq 1$, as $w$ is not a fixed element of $\tau, q=p$ is the common order of $v$ and $\tau$.

From $P=P^{p}$ we deduce the existence of an element $u$ in $P$ such that $u^{p}=w$. Let $r=u^{r-1}$. Then

$$
w v=w^{\tau}=u^{p r}=u^{\tau p}=u^{p} \boldsymbol{r}^{p} \text { or } \boldsymbol{r}^{p}=v .
$$

Since $v$ has order $p, r$ has order $p^{2}$; and consequently $r$ is a fixed element of $\tau$. Therefore

$$
u^{r^{i}}=u r^{i} \text { for every positive } j \text {. }
$$

Since $p$ is the order of $\tau$, we find that

$$
u=u^{\tau^{p}}=u r^{p} \text { or } r^{p}=1 ;
$$


this contradicts the fact, established before, that the order of $r$ is $p^{2}$. This contradiction proves $\sigma=1$, as we desired to show.

REMARK 1. If $P$ is the group of type $2^{\infty}$ and $\sigma$ the automorphism of $P$ which maps every element in $P$ onto its inverse, then the order of $\sigma$ is 2 . The first and third hypotheses of our lemma are satisfied by $\sigma$, the second one only in the weaker form that elements of order $p[=2]$ are fixed elements. Thus we cannot, in general, weaken the second hypothesis.

REMARK 2. If $P$ is a group of type $p^{\infty}$ and $\sigma$ the automorphism of $P$ which maps $x$ in $P$ onto $x^{p^{2}+1}$, then the first and second hypotheses of our lemma are satisfied, but not the third one. Thus the third hypothesis is indispensable.

For our application it will be convenient to derive from our lemma the following

Corollary. If the minimum condition is satisfied by the subgroups of the abelian group $A$, then there exists a positive integer $n=n(A)$ with the following property:

If the order of the automorphism $\sigma$ of $A$ is finite, and if $x^{n}=1$ implies $x^{\sigma}=x$, then $\sigma=1$.

Proof. Since the minimum condition is satisfied by the subgroups of $A$, $A$ is a torsion group; and $A$ is, as an abelian torsion group, the direct product of its primary components $A(p)$. The minimum condition implies that only a finite number of the $A(p)$ is different from 1.

Since the minimum condition is satisfied by the subgroups of the abelian $p$-group $A(p)$, it is the product of a finite group $F(p)$ and of a characteristic subgroup $B(p)$ satisfying $B(p)=B(p)^{p}$-clearly $B(p)$ is also a characteristic subgroup of $A$. We denote by $k(p)$ the maximum order of elements in $F(p)$; and we let $n(p)$ be the maximum of $p^{2}$ and $k(p)$. Finally we denote by $n=n(A)$ the product of the finitely many $n(p)$ with $A(p) \neq 1$.

Suppose now that $\sigma$ is an automorphism of $A$, that the order of $\sigma$ is finite, and that $x^{n}=1$ implies $x^{\sigma}=x$. It is clear that $\sigma$ induces automorphisms in the characteristic subgroups $A(p)$ and $B(p)$ of $A$. From our choice of $n$ and $n(p)$ it follows that every element in $F(p)$ is a fixed element of $\sigma$. Since $p^{2}$ is a factor of $n(p)$, we deduce from the lemma that every element in $B(p)$ is a fixed element of $\sigma$. Hence every element in $A(p)=F(p) B(p)$ is a fixed element of $\sigma$; and consequently $\sigma=1$.

3. We are now ready to deduce (i) from (ii). Accordingly we assume in this section that $G / Z(G)$ is finite and that the minimum condition is satisfied by the subgroups of $Z(G)$.

Consider now a torsion group $\Gamma$ of automorphisms of $G$. We denote by $\Delta$ the totality of automorphisms in $\Gamma$ which leave invariant every element in $Z(G)$. It is clear that $\Delta$ is a normal subgroup of $\Gamma$ and that $\Gamma / \Delta$ is essentially the same as the group $\Gamma^{*}$ of automorphisms of $Z(G)$ which are induced in $Z(G)$ by automorphisms in $\Gamma$. 
Since the minimum condition is satisfied by the subgroups of the abelian group $Z(G)$, there exists a positive integer $n$ with the following property:

(+) The automorphism $\sigma$ of $Z(G)$ is equal to 1 if, and only if, the order of $\sigma$ is finite and $x^{n}=1$ implies $x^{\sigma}=x$.

[The existence of $n$ is a consequence of $\$ 2$, Corollary.] We denote by $N$ the totality of elements $x$ in $Z(G)$ which satisfy $x^{n}=1$. This is a characteristic subgroup of $Z(G)$ and $G ; N$ is finite, since the minimum condition is satisfied by the subgroups of the abelian group $Z(G)$. Since $\Gamma$ is a torsion group, so is its homomorphic image $\Gamma^{*}$, and now we deduce from $(+)$ that $\Gamma^{*}$ is essentially the same as the group of automorphisms of $N$ which are induced in $N$ by automorphisms in $\Gamma^{*}$. But groups of automorphisms of finite groups are finite. Hence $\Gamma^{*}$ is finite, and this implies the finiteness of $[\Gamma: \Delta]$.

Denote by $\Sigma$ the totality of automorphisms $\sigma$ in $\Delta$ which satisfy

$$
x^{\sigma} \equiv x \text { modulo } Z(G)
$$

for every $x$ in $G$. It is fairly clear that $\Sigma$ is a normal subgroup of $\Delta$; and that $\Delta / \Sigma$ is essentially the same as the group of automorphisms of $G / Z(G)$ which are induced by automorphisms in $\Delta$. Since $G / Z(G)$ is finite, so are the groups of automorphisms of $G / Z(G)$. Hence $[\Delta: \Sigma]$ is finite.

If $\sigma$ is an automorphism in $\Sigma$, then every element in $Z(G)$ and every element in $G / Z(G)$ is a fixed element of $\sigma$. If $g$ is an element in $G$ and $z$ an element in $Z(G)$, then

$$
g^{\sigma-1}=(g z)^{\sigma-1}
$$

is an element in $Z(G)$. Consequently $X^{\sigma-1}$ is a well determined element in $Z(G)$ for every coset $X$ in $G / Z(G)$. If $m$ is the finite order of $G / Z(G)$ and $g$ is an element in $G$, then $g^{m}$ belongs to $Z(G)$. Hence

$$
1=\left(g^{m}\right)^{\sigma-1}=g^{m \sigma} g^{-m}=g^{\sigma m} g^{-m}=\left(g^{\sigma-1} g\right)^{(m)} g^{-m}=g^{(\sigma-1) m},
$$

since $g^{\sigma-1}$ belongs to $Z(G)$. Hence every $X^{\sigma-1}$ has an order dividing $m$. Denote by $M$ the totality of elements $z$ in $Z(G)$ satisfying $z^{m}=1$. Since the minimum condition is satisfied by the subgroups of the abelian group $Z(G), M$ is a finite subgroup of $Z(G)$. Thus we see that $\Sigma$ is essentially the same as a set of mappings [actually a group of homomorphisms] of $G / Z(G)$ into $M$. Since $G / Z(G)$ and $M$ are both finite, there exists only a finite number of mappings of $G / Z(G)$ into $M$. Hence $\Sigma$ is finite.

Since $[\Gamma: \Delta],[\Delta: \Sigma]$ and $\Sigma$ are all finite, $\Gamma$ is finite. Hence (i) is a consequence of (ii); and this completes the proof of the equivalence of properties (i) and (ii).

4. In this section we are going to prove the equivalence of conditions (ii), (iii) and (iv). If $G / Z(G)$ is finite, then $[G, G]$ is likewise finite; see, for instance, Baer [4, p. 163, Zusatz]. If furthermore the minimum condition is 
satisfied by the subgroups of $Z(G)$, then it is satisfied by the subgroups of $G$ and hence by the subgroups of $G /[G, G]$. Thus (iii) is a consequence of (ii). It is almost obvious that (iv) is a consequence of (iii).

Assume now the validity of (iv). There exists, as in every group, a maximal abelian subgroup $A$ of $G$. Since the minimum condition is satisfied by the subgroups of $A$, there exists a uniquely determined subgroup $B$ of $A$ such that

$$
A / B \text { is finite and } B=B^{n} \text { for every positive integer } n \text {. }
$$

If $g$ is any element in $G$, then $g$ possesses only a finite number of conjugate elements in $G$ [by (iv)]. The centralizer $C(g)$ of $g$ in $G$ has therefore finite index $[G: C(g)]$ in $G$. This implies the finiteness of the index $[B: B \cap C(g)]$. But $B$ does not possess proper subgroups of finite index. Hence $B=B \cap C(g)$ or $B \leqq C(g)$ for every $g$ in $G$; and this implies

$$
B \leqq Z(G) \text {. }
$$

If $x$ is an element in the centralizer of $A$ in $G$, then $\{A, x\}$ is an abelian subgroup of $G$, since $A$ is abelian; and now we deduce $A=\{A, x\}$ from the maximality of $A$. Hence $x$ belongs to $A$; and this proves that $A$ is equal to its centralizer in $G$. Since $A / B$ is finite, there exists a finite subset $F$ of $A$ such that $A=\{B, F\}$. Since $B$ is part of the center $Z(G)$ of $G$, an element belongs to the centralizer of $A$ if, and only if, it belongs to the centralizer of $F$. Hence

$$
A=\bigcap_{f \text { in } F} C(f) .
$$

We have pointed out before that each of the finitely many indices $[G: C(f)]$ is finite. By Poincaré's Theorem, the index $[G: A]$ is finite too. Since $[A: B]$ is finite, it follows that $[G: B]$ is finite. Since $B \leqq Z(G)$, this shows the finiteness of $G / Z(G)$; and since $Z(G)$ is abelian, the minimum condition is satisfied by its subgroups [by (iv)]. Thus (ii) is a consequence of (iv); and this completes the proof of the equivalence of conditions (i) to (iv).

5. In this section we are going to prove the equivalence of condition (v) with conditions (i) to (iv) [whose equivalence has already been verified]. Assume firstly the validity of conditions (i) to (iv). Then every homomorphic image $J \neq 1$ of $G$ likewise satisfies condition (ii). Thus $J / Z(J)$ is finite; and the minimum condition is satisfied by the subgroups of $Z(J)$. This implies in particular that $Z(J)$ contains only a finite number of elements of squarefree order. If $x$ and $y$ are elements in $J$ such that $x^{n}=y^{n}=1$ and such that $x \equiv y$ modulo $Z(J)$, then there exists an element $z$ in $Z(J)$ such that $x=z y$; and we have $1=x^{n}=z^{n} y^{n}=z^{n}$; and now it is clear that $J$ contains only a finite number of elements of squarefree order. Since $J$ is a torsion group and $J \neq 1, J$ contains elements of order a prime. Hence (v) is satisfied by $G$.

Assume conversely the validity of (v). It is known that a finite class of conjugate elements of finite order generates a finite normal subgroup; see 
Neumann [1, p. 186, 5.22, Corollary]. Thus (v) implies the following stronger property:

$\left(\mathrm{v}^{*}\right)$ If $J \neq 1$ is a homomorphic image of $G$, then the elements of squarefree order in $J$ generate a finite normal subgroup $Q(J) \neq 1$ of $J$.

There exist normal subgroups of $G$ which are torsion groups; and among these there exists a maximal one, say $N$. If $N$ were different from $G$, then $Q(G / N)$ would be a finite normal subgroup, not 1 , of $G / N$; and this would lead to an obvious contradiction because of the maximality of $N$. Hence $G=N$ is a torsion group.

Consider now an abelian subgroup $A$ of $G$. Since $\dot{G}$ is a torsion group, $A$ is an abelian torsion group; and since $G$ contains only finitely many elements of squarefree order, so does $A$. Hence the minimum condition is satisfied by the subgroups of $A$; and consequently the minimum condition is satisfied by the abelian subgroups of $G$.

We define inductively an ascending chain of characteristic subgroups $Q_{i}(G)$ of $G$ by the rules:

$$
Q_{0}(G)=1, \quad Q_{i+1}(G) / Q_{i}(G)=Q\left[G / Q_{i}(G)\right] .
$$

By an obvious inductive argument one deduces from $\left(\mathrm{v}^{*}\right)$ that every $Q_{i}(G)$ is finite; and that $Q_{i}(G)$ contains every element in $G$ whose order is not divisible by an $(i+1)$ st power [of a prime]. Since $G$ has been shown to be a torsion group, we see that every element in $G$ belongs to at least one of these finite normal subgroups $Q_{i}(G)$ of $G$. This implies in particular that classes of conjugate elements in $G$ are finite. Hence (iv) is a consequence of (v); and this completes the proof of the equivalence of conditions (i) to (v).

6. As an application of our principal theorem we prove the following

COROLLARY. Torsion groups with finite automorphism group are finite.

Proof. If $G$ is a torsion group whose group of automorphisms is finite, then an immediate application of our principal theorem shows that $G / Z(G)$ is finite and that $G$ contains only a finite number of elements of squarefree order. We denote by $k$ the product of the finitely many primes that are orders of elements in $G$. As in $\$ 1$ we show the existence of a finite subgroup $S$ of $G$ such that $G=Z(G) S$; and we denote by $m$ the order of $S$. Then $1+k m$ is prime to the order of every element in $G$ so that the mapping $\sigma$ defined by $x^{\sigma}=x^{1+k m}$ for every $x$ in $G$ is a one to one mapping of $G$ upon itself. But $\sigma$ leaves invariant every element in $S$ and $\sigma$ induces an automorphism in the abelian subgroup $Z(G)$. Consequently $\sigma$ is an automorphism of $G$, and this automorphism has, by hypothesis, finite order $j$. Consequently $x=x^{(1+k m)^{i}}$ for every $x$ in $G$ so that the orders of the elements in $G$ are bounded. Since the minimum condition is satisfied by the subgroups of $Z(G)$ and since the orders of the elements in $Z(G)$ are bounded, the abelian group $Z(G)$ is finite. Since $Z(G)$ and $G / Z(G)$ are finite, $G$ is finite, as we wanted to show. 
REMARK. If we form the direct product of a finite group and an infinite cyclic group, then we obtain an infinite group whose automorphism group is finite but which, naturally, is not a torsion group.

The author is indebted to the referee for the following application of the preceding corollary.

A group $G$ is finite if it possesses only finitely many endomorphisms.

Proof. If the set of endomorphisms of $G$ is finite, then the group of inner automorphisms of $G$ is finite, and this implies the finiteness of $G / Z(G)$. If $j=[G: Z(G)]$, then the transfer of $G$ into $Z(G)$ is an endomorphism of $G$ mapping every element upon its $j$ th power. Since there exists only a finite number of endomorphisms of $G$, there exists a positive integer $n$ such that $g^{j^{n}}=g^{j^{n+1}}$ for every $g$ in $G$. If $j \neq 1$, then this implies that $G$ is a torsion group; torsion groups possessing but a finite number of automorphisms are finite by the corollary. If $j=1$, then $G=Z(G)$ is an abelian group, and mapping every element in $G$ upon its $n$th power is, for every integer $n$, an endomorphism. But $G$ possesses only a finite number of endomorphisms, and consequently there exist two different integers $h$ and $k$ such that $g^{h}=g^{k}$ for every $g$ in $G$. Again we see that $G$ is a torsion group and that $G$ is finite by the corollary.

7. Our principal theorem will now be used to obtain a characterization of a more comprehensive class of groups, namely the groups with minimum condition and an abelian subgroup of finite index.

Propositron. The following properties of the group $G$ are equivalent.

(1) The minimum condition is satisfied by the normal subgroups of $G$ and there exists an abelian subgroup of finite index in $G$.

(2) The minimum condition is satisfied by the abelian subgroups of $G$ and there exists a maximal abelian subgroup of $G$ which possesses only a finite number of conjugates in $G$.

(3) The minimum condition is satisfied by the abelian subgroups of $G$ and every infinite homomorphic image $H$ of $G$ contains an element $x \neq 1$ which possesses only a finite number of conjugates in $H$.

(4) (a) $G$ is a torsion group.

(b) Simple subgroups of homomorphic images of $G$ are finite.

(c) If $H$ is an infinite homomorphic image of $G$, and if 1 is the intersection of the subgroups of finite index in $H$, then there exists a minimal normal subgroup of $H$.

(d) Every torsion group of automorphism classes of every subgroup of $G$ is finite.

REMARK 1 . If the minimum condition is satisfied by the normal subgroups of $G$, then the intersection $J(G)$ of all the subgroups of finite index in $G$ is a characteristic subgroup of $G$ whose index $[G: J(G)]$ is finite; see, for instance, Baer $[3$, p. $3, \S 2$, Proposition 2]. Thus condition (1) implies the existence of an abelian characteristic subgroup of finite index in $G$. 
REMARK 2. By an obvious combination of the equivalent conditions (1) and (2) one sees that the minimum condition is satisfied by the subgroups of groups with properties (1), (2).

REMARK 3. It is clear that every group satisfying condition (ii) of $\S 0$, Theorem likewise satisfies the above condition (1). That the converse is not true, one sees from easily constructed examples; such an example one obtains by adjoining to an infinite abelian group $A$ with minimum condition an element $b$ satisfying $(b a)^{2}=1$ for every $a$ in $A$.

REMARK 4. As has been pointed out in Remark 2 the class of groups under consideration is the class of all groups with minimum condition possessing an abelian subgroup of finite index. It is clear that this class contains with any group $G$ every subgroup and homomorphic image of $G$.

REMARK 5. It seems to be difficult to assess the strength of condition (4.d). The reader may be reminded of the fact that every automorphism of the infinite symmetric group is inner, i.e. its group of automorphism classes equals 1 , though we cannot claim anything like this of the subgroups of this symmetric group.

REMARK 6. The conditions of the principal theorem often require that every subgroup [every element] has a certain property [like possession of only a finite number of conjugates] whereas the corresponding conditions of our proposition require only the existence of at least one subgroup [at least one element] with the corresponding property. For instance, condition (ii) of $\S 0$, Theorem implies that every subgroup possesses only a finite number of conjugates whereas condition (2) of our proposition requires the existence of at least one maximal abelian subgroup possessing only a finite number of conjugates. That we cannot claim more may be seen from the following example: Let $A$ be an infinite abelian group with minimum condition and without elements of order 2; and denote by $G$ the group arising from $A$ by adjunction of an element $b$, subject to the condition $(b a)^{2}=1$ for every $a$ in $A$. Then $G$ meets all our requirements; but the subgroups $\{b a\}$ for $a$ in $A$ form an infinite class of conjugate maximal abelian subgroups of $G$.

REMARK 7. If $H$ is a homomorphic image of a group, satisfying the equivvalent conditions (1) to (4), then either $H$ is finite or else the abelian characteristic subgroup $J(H)$ of finite index in $H$ is infinite; see Remark 1. Since the minimum condition is satisfied by the subgroups of the abelian group $J(H)$, the elements of squarefree order in $J(H)$ form a finite characteristic subgroup of $H$ which is different from 1 whenever $J(H) \neq 1$, e.g. whenever $H$ is infinite. Thus we see that every group $G$ with the equivalent properties (1) to (4) has the following property:

every homomorphic image $H \neq 1$ of $G$ contains a finite characteristic subgroup $\neq 1$.

This improves upon (3).

8. In this section we are going to prove the equivalence of properties (1) 
and (2). If property (1) is satisfied by the group $G$, then there exists an abelian subgroup of finite index in $G$; and this implies the existence of a maximal abelian subgroup $A$ of finite index [G:A] in $G$. But the normalizer of $A$ in $G$ contains $A$ and has therefore finite index too so that $A$ possesses only a finite number of conjugate subgroups in $G$. It is clear furthermore that the intersection $J(G)$ of all the subgroups of finite index in $G$ is abelian. But the minimum condition is satisfied by the subgroups of $G$ whenever $J(G)$ is abelian and the minimum condition is satisfied by the normal subgroups of $G$; see Baer [3, p. 15-16, $\S 5$, Theorems 2 and 4]. Hence (2) is a consequence of (1).

Assume conversely the validity of (2). Then there exists a maximal abelian subgroup $A$ of $G$ which possesses only a finite number of conjugate subgroups in $G$. This latter property is equivalent to the fact that the index $[G: B]$ of the normalizer $B$ of $A$ in $G$ is finite. Since $A$ is a maximal abelian subgroup of $G, A$ is equal to its own centralizer in $G$. Hence $B / A$ is essentially the same as a group of automorphisms of $A$. Since the minimum condition is satisfied by the abelian subgroups of $G, G$ is a torsion group. Thus $B / A$ is a torsion group of automorphisms of $A$ and the minimum condition is satisfied by the subgroups of the abelian group $A$. We apply our principal theorem to see that $B / A$ is finite. Since the indices $[G: B]$ and $[B: A]$ are finite, $[G: A]$ is finite. Since the minimum condition is satisfied by the subgroups of $A$ and since $[G: A]$ is finite, the minimum condition is satisfied by the subgroups of $G$. Hence (1) is a consequence of (2).

9. In this section we show the equivalence of (3) and the equivalent conditions (1) and (2). Assume first the validity of the equivalent conditions (1) and (2). Then we deduce from (2) the validity of the first half of condition (3). If $H$ is an infinite homomorphic image of $G$, then the intersection $J(H)$ of all the subgroups of finite index in $H$ is abelian and $[H: J(H)]$ is finite; see $\S 7$, Remark 1 . Since the minimum condition is satisfied by the subgroups of $J(H), J(H)$ contains only a finite number of elements of squarefree order. Since $H$ is infinite and the index of $J(H)$ is finite, $J(H) \neq 1$. Hence $J(H)$ contains elements of prime number order; their number is finite and they form complete classes of conjugate elements in $H$. Thus (3) is a consequence of (1) and (2).

Next we assume the validity of condition (3). It is clear then that $G$ is a torsion group. We prove first the following fact which will be used variously in the course of our proof.

(a) If $N$ is a normal subgroup of the subgroup $S$ of $G$, and if $N$ and $S / N$ are both abelian, then there exists an abelian subgroup of finite index in $S$ and the minimum condition is satisfied by the subgroups of $S$.

Since $N$ is an abelian subgroup of $S$, there exists a maximal abelian subgroup $A$ of $S$ which contains $N$. Since $S / N$ is abelian, $A / N$ is a normal subgroup of $S / N$ and hence $A$ is a normal subgroup of $S$. Since $A$ is a maximal 
abelian subgroup of $S, A$ is its own centralizer in $S$. Hence $S / A$ is essentially the same as a group of automorphisms of $A$. Since $S / A$ is a torsion group and since the minimum condition is satisfied by the subgroups of the abelian group $A$ [by (3) ], $S / A$ is finite as a consequence of our principal theorem. Since $S / A$ is finite and the minimum condition is satisfied by the subgroups of $A$, the minimum condition is satisfied by the subgroups of $S$, proving (a).

There exists among the abelian normal subgroups of $G$ a maximal one, say $M$. It is an immediate consequence of (a) that the minimum condition is satisfied by the abelian subgroups of $G / M$. We form the totality $F=F(G / M)$ of elements in $G / M$ which possess only a finite number of conjugates in $G / M$. We have shown elsewhere that $F$ is a characteristic subgroup of $G / M$; see Baer [2, p. 1023, §2, Proposition 1]. Since every element in $F$ possesses only a finite number of conjugates in the group $F$, and since the minimum condition is satisfied by the abelian subgroups of $F$, we may apply our principal theorem on $F$ to see that $F / Z(F)$ is finite.

Denote by $H$ and $K$ the uniquely determined subgroups of $G$ which contain $M$ and satisfy $F=H / M$ and $Z(F)=K / M$. Since $F$ and $Z(F)$ are characteristic subgroups of $G / M, K$ and $H$ are normal subgroups of $G$. It is clear furthermore that $H / K \simeq F / Z(F)$ is finite and that $K / M$ is abelian. Since $M$ and $K$ are normal subgroups of $G$, the centralizer $L$ of $M$ in $K$ is a normal subgroup of $G$. Since $M$ is abelian and $K / L$ is a torsion group of automorphisms of $M$, we deduce as usual from our principal theorem that $K / L$ is finite. Since $M$ and the subgroup $L / M$ of $K / M$ are abelian, we deduce from (a) [and $\S 7$, Remark 1] the existence of an abelian characteristic subgroup $J(L)$ of finite index in $L$. Since $J(L)$ is a characteristic subgroup of the normal subgroup $L$ of $G, J(L)$ is a normal subgroup of $G$. Since $M$ is part of the center of $L$ and $J(L)$ is an abelian subgroup of $L, M J(L)$ is an abelian normal subgroup of $G$. Since $M$ is a maximal abelian normal subgroup of $G$, we find that $M=M J(L)$ or $J(L) \leqq M$. Hence $[L: M]$ is finite. Thus the indices $[H: K]$, $[K: L]$, and $[L: M]$ are finite; and this implies the finiteness of $F=H / M$.

It is noteworthy that the second part of condition (3) has not been used up till now. Assume by way of contradiction that $G / M$ is infinite. Since $H / M$ is finite, this implies the infinity of $G / H$. Consequently there exists [by (3)] an element $x \neq 1$ in $G / H$ which possesses only a finite number of conjugates in $G / H$. Let $y$ be some element in $G$ such that $x=H y$. Since $H / M$ is finite, and since $x$ possesses only a finite number of conjugates in $G / H, M y$ possesses only a finite number of conjugates in $G / M$. It follows from the definition of $F$ that $M y$ belongs to $F$. Hence $y$ is in $H$ so that $1=x \neq 1$; this is the desired contradiction. Consequently $G / M$ is finite. Since the minimum condition is satisfied by the subgroups of the abelian group $M$, the minimum condition is satisfied by the subgroups of $G$; thus we see that (1) is a consequence of (3).

10. In this section we are going to show that (4) is a consequence of the equivalent conditions (1) to (3). In the presence of these conditions the 
minimum condition is satisfied by subgroups of $G$ and $G$ possesses an abelian characteristic subgroup of finite index. Clearly the same is true of all the subgroups of homomorphic images of $G$; and this puts into evidence the validity of conditions (4.a) to (4.c).

Consider now some subgroup $S$ of $G$. Then the minimum condition is satisfied by the subgroups of $S$ and the intersection $J(S)$ of all the subgroups of finite index in $S$ is an abelian characteristic subgroup of finite index in $S$; cf. §7, Remark 1.

Every group of automorphism classes of $S$ has the form $\phi / \Gamma$ where $\phi$ is a group of automorphisms of $S$ and where $\Gamma$ is the group of inner automorphisms of $S$. Since $S$ is a torsion group, and since $\Gamma \simeq S / Z(S), \Gamma$ is a torsion group. Hence $\phi / \Gamma$ is a torsion group if, and only if, $\phi$ is a torsion group; and this is what we are going to assume in the sequel.

Denote by $\Delta$ the group of all those automorphisms in $\phi$ which leave invariant every element in $J(S)$. Since $J(S)$ is a characteristic subgroup of $S$, $\Delta$ is a normal subgroup of $\phi$ and $\phi / \Delta$ is essentially the same as the group of automorphisms of $J(S)$ which are induced in $J(S)$ by automorphisms in $\phi$. Clearly this is a torsion group of automorphisms. Since the minimum condition is satisfied by the subgroups of the abelian group $J(S)$, by our principal theorem $\phi / \Delta$ is finite.

Denote by $\Sigma$ the totality of automorphisms $\sigma$ in $\Delta$ which satisfy

$$
g^{\sigma} \equiv g \text { modulo } J(S)
$$

for every $g$ in $S$. Since $J(S)$ is a characteristic subgroup of $S, \Sigma$ is a normal subgroup of $\Delta$ and $\Delta / \Sigma$ is essentially the same as a group of automorphisms of the finite group $S / J(S)$. Hence $\Delta / \Sigma$ is finite too.

Denote by $n$ the finite order of $S / J(S)$ and by $N$ the totality of elements $x$ in $J(S)$ which satisfy $x^{n}=1$. Since the minimum condition is satisfied by the subgroups of the abelian group $J(S), N$ is a finite characteristic subgroup of $J(S)$. Denote by $\Sigma^{*}$ the totality of automorphisms $\sigma$ in $\Sigma$ which satisfy

$$
s^{\sigma} \equiv s \text { modulo } N \text { for every } s \text { in } S .
$$

It is clear that $\Sigma^{*}$ is a normal subgroup of $\Sigma$, and that therefore every element in $\Sigma^{*}$ leaves invariant every element in $J(S)$. Thus $s^{\sigma-1}=[J(S) s]^{\sigma-1}$ for every $s$ in $S$ and every $\sigma$ in $\Sigma^{*}$. Hence $\Sigma^{*}$ is essentially the same as a set of singlevalued mappings of the finite group $S / J(S)$ into the finite group $N$; and consequently $\Sigma^{*}$ is finite.

Consider now an automorphism $\sigma$ in $\Sigma$. Then $\sigma$ induces the identity both in $J(S)$ and in $S / J(S)$. If $X$ is a coset in $S / J(S)$, then $X^{\sigma-1}$ is a well determined element in $J(S)$. Since $J(S)$ is abelian and $S / J(S)$ is finite, we may form the product

$$
s=\prod X^{\sigma-1}
$$


where the product ranges over all the $X$ in $S / J(S)$. Since $J(S)$ is an abelian group without proper subgroups of finite index [as every subgroup of finite index in $J(S)$ has finite index in $S$ ] we have $J(S)=J(S)^{n}$; and consequently there exists an element $t$ in $J(S)$ such that $t^{n}=s$. Denote by $\tau$ the inner automorphism of $S$ which is induced by $t$ so that $x^{\tau}=t^{-1} x t$ for every $x$ in $S$. If $y$ is an element in $S$, then

$$
y^{\sigma \tau-1}=t^{-1} y^{\sigma} t y^{-1}=t^{-1} y^{\sigma-1} y t y^{-1}=y^{\sigma-1} t^{-1} y t y^{-1}=y^{\sigma-1} y^{\tau-1},
$$

since $y^{\sigma-1}$ and $t$ belong both to the abelian group $J(S)$; and we note that $y^{\tau-1}$ belongs to $J(S)$, since $t$ belongs to the characteristic subgroup $J(S)$ of $S$. Thus $y^{\sigma \tau-1}$ belongs likewise to $J(S)$. Since $y^{\sigma-1}$ and $t$ belong to the abelian subgroup $J(S)$, we find furthermore that

$$
\begin{aligned}
{\left[y^{\sigma \tau-1}\right]^{n} } & =\left[y^{\sigma-1}\right]^{n}\left[y^{\tau-1}\right]^{n}=y^{(\sigma-1) n} t^{-n} y t^{n} y^{-1}=y^{(\sigma-1) n} s^{-1} y s y^{-1} \\
& =y^{(\sigma-1) n} \prod\left[X^{1-\sigma} y X^{\sigma-1} y^{-1}\right] \\
& =\prod\left[X^{1-\sigma} y^{\sigma} X^{\sigma-1} y^{-1}\right]=\prod X^{1-\sigma} \prod(y X)^{\sigma-1}=1
\end{aligned}
$$

where all the products have to be taken over the $n$ cosets $X$ in $S / J(S)$. Thus we have shown that $y^{\sigma \tau-1}$ belongs to $N$ for every $y$ in $S$ or

$$
y^{\sigma \tau} \equiv y \text { modulo } N \text { for every } y \text { in } S .
$$

If in particular $x$ is an element in $J(S)$, then $x^{\sigma \tau}=t^{-1} x^{\sigma} t=x$, since $x$ and $t$ are both in the abelian group $J(S)$. Thus we have shown that $\sigma \tau$ belongs to $\Sigma^{*}$. Hence $\Sigma \leqq \Sigma^{*} \Gamma$. Since $\Sigma^{*} \leqq \Sigma$, it follows that $\Sigma \Gamma=\Sigma^{*} \Gamma$ and that consequently

$$
\Sigma \Gamma / \Gamma=\Sigma^{*} \Gamma / \Gamma \simeq \Sigma^{*} /\left[\Sigma^{*} \cap \Gamma\right]
$$

is finite.

If we remember finally that $\phi / \Delta$ and $\Delta / \Sigma$ are finite, it becomes clear that $\phi / \Gamma$ is finite, as we wanted to show.

11. Assume now that condition (4) is satisfied by $G$. We are going to prove that the equivalent properties (1) to (3) hold in $G$. To do this we shall first substitute for conditions (4.a) and (4.d) some weaker and handier conditions.

If $A$ is an abelian subgroup of $G$, then $A$ is a torsion group [by (4.a)] and every torsion group of automorphisms of $A$ is finite [by (4.d)]. We apply our principal theorem to see that the minimum condition is satisfied by the subgroups of $A$. Hence

$\left(a^{\prime}\right)$ the minimum condition is satisfied by the abelian subgroups of $G$.

If $S$ is a subgroup of $G$, then we denote by $C(S<G)$ the centralizer and by $N(S<G)$ the normalizer of $S$ in $G$. It is clear that $S C(S<G)$ is a normal subgroup of $N(S<G)$ and that an element in $G$ induces an inner automorphism in $S$ if, and only if, it belongs to $S C(S<G)$. Thus $N(S<G) / S C(S<G)$ is essentially the same as a group of automorphism classes of $S$; and this group 
of automorphism classes of $S$ is a torsion group, since $G$ is a torsion group [by (4.a)]. We deduce from (4.d) that

$\left(\mathrm{d}^{\prime}\right) \quad N(S<G) / S C(S<G)$ is finite for every subgroup $S$ of $G$.

Suppose next that $T$ is a normal subgroup of the subgroup $S$ of $G$, that $S / T$ is abelian and that $T$ contains an abelian subgroup of finite index. Since the minimum condition is satisfied by the abelian subgroups of $T$ [by $\left(a^{\prime}\right)$ ], the minimum condition is satisfied by the subgroups of $T$. The intersection $J(T)$ of all the subgroups of finite index in $T$ is therefore an abelian subgroup of finite index in $T$; see $\S 7$, Remark 1 . Since $J(T)$ is a characteristic subgroup of the normal subgroup $T$ of $S, J(T)$ is a normal subgroup of $S$. Next we note that the minimum condition is satisfied by the abelian subgroups of $S$ [by $\left(\mathrm{a}^{\prime}\right)$ ]. Consider now a normal subgroup $N \neq S$ of $S$. If firstly $T \leqq N$, then $S / N$ is abelian and every element in $S / N$ possesses only a finite number of conjugatesin $S / N$ [namelyone]. If $J(T) \leqq N$, but $T \leqq N$, then $N T / N \simeq T /(N \cap T \$)$ is finite as a homomorphic image of $T / J(T)$; and so every element in the finite normal subgroup $N T / N \neq 1$ of $S / N$ possesses only a finite number of conjugates in $S / N$. If finally $-J(T) \leq N$, then $N J(T) / N \simeq J(T) /[N \cap J(T)]$ is an abelian normal subgroup, not 1 , of $S / N$; and the minimum condition is satisfied by the subgroups of $N J(T) / N$, since the minimum condition is satisfied by the subgroups of $J(T)$ [by $\left.\left(\mathrm{a}^{\prime}\right)\right]$. Consequently there exists only a finite number of elements of any given order in $N J(T) / N$ so that elements in $N J(T) / N$ possess only a finite number of conjugates in $S / N$. Thus we have verified the validity of (3) in $S$. But (1), (2), and (3) are equivalent; thus we have shown the following fact:

(a") If $T$ is a normal subgroup of the subgroup $S$ of $G$, if $T$ contains an abelian subgroup of finite index, and if $S / T$ is abelian, then the minimum condition is satisfied by the subgroups of $S$.

Among the abelian normal subgroups of $G$ there exists a maximal one, say $M$. The centralizer of $M$ in $G$ is a normal subgroup of $G$ which contains $M$. By $\left(\mathrm{d}^{\prime}\right)$ therefore

(e.1) $G / C(M<G)$ is finite.

Denote by $P / M$ the product of all the finite normal subgroups of $G / M$. It is clear that $P$ is a normal subgroup of $G$; and, by $\left(\mathrm{a}^{\prime}\right)$, the minimum condition is satisfied by the abelian subgroups of $P$. If $N \neq P$ is a normal subgroup of $P$ which contains $M$, then there exists a finite normal subgroup $X / M$ of $G / M$ which is not part of $N / M[<P / M]$. Thus $N X / N \simeq X /(X \cap N)$ is a homomorphic image of the finite group $X / M$ and this finite normal subgroup, not 1 , of $P / N$ contains an element, not 1 , which possesses only a finite number of conjugates in $P / N$. If next the normal subgroup $N$ of $P$ does not contain $M$, then $N M / N \simeq M /(N \cap M)$ is a homomorphic image of the abelian subgroup $M$ of $G$. By $\left(\mathrm{a}^{\prime}\right)$ the minimum condition is satisfied by the subgroups of $M$; and thus $N M / N$ is an abelian normal subgroup of $P / M$ which possesses only a finite number of elements of any given order. Hence $N M / N$ con- 
tains elements, not 1 , which possess only a finite number of conjugates in $P / N$. We have verified the validity of (3). Because of the equivalence of conditions (1), (2), and (3) we may now deduce from $\$ 7$, Remark 1 , that the intersection $J(P)$ of all the subgroups of finite index in $P$ is a characteristic and abelian subgroup of $P$ whose index $[P: J(P)]$ is finite. As a characteristic subgroup of a normal subgroup $J(P)$ is a normal subgroup of $G$. The product $M[J(P) \cap C(M<G)]$ of two abelian normal subgroups each of which is part of the centralizer of the other is a normal and abelian subgroup of $G$. But $M$ is a maximal abelian normal subgroup of $G$; and consequently we have

$$
M=M[J(P) \cap C(M<G)] \text { or } J(P) \cap C(M<G) \leqq M .
$$

Since

$$
J(P) /[J(P) \cap C(M<G)] \simeq C(M<G) J(P) / C(M<G) \leqq G / C(M<G)
$$

is finite by (e.1), and $P / J(P)$ has been shown to be finite before, $P /[J(P)$ $\cap C(M<G)]$ is finite too; and consequently

(e.2) $P / M$ is finite.

If $X / P$ is a finite normal subgroup of $G / P$, then $X / M$ is, by (e.2), a finite normal subgroup of $G / M$. By the definition of $P$ we have therefore $P \leqq X \leqq P$ or $P=X$ so that

(e.3) 1 is the only finite normal subgroup of $G / P$.

If $A / P$ is an abelian subgroup of $G / P$, then we note that $P$ contains the abelian normal subgroup $M$ whose index $[P: M]$ is finite by (e.2). It follows from $\left(\mathrm{a}^{\prime \prime}\right)$ that the minimum condition is satisfied by the subgroups of $A$ and hence also by those of $A / P$. If $A$ is in particular a normal subgroup of $G$, then the elements of squarefree order in $A / P$ form a characteristic subgroup of $A / P$ and hence a normal subgroup of $G / P$ which is finite, since the minimum condition is satisfied by the subgroups of the abelian group $A / P$. By (e.3) this normal subgroup equals 1 ; and so $A / P=1$. Thus we have shown the following facts

(e.4) The minimum condition is satisfied by the abelian subgroups of $G / P$ and 1 is the only abelian normal subgroup of $G / P$.

Assume now by way of contradiction that $P<G$. By (e.3), $G / P$ is infinite. Denote by $J(G / P)$ the intersection of all the subgroups of finite index in $G / P$. We distinguish two cases.

CASE 1. $J(G / P)=1$. In this case there exists, by (4.c), a minimal normal subgroup $W / P$ of $G / P$. Because of $J(G / P)=1$ there exists a subgroup $S$ of finite index in $G / P$ which does not contain $W / P$. By Poincaré's Theorem there exists a normal subgroup $V / P$ of $G / P$ which is part of $S$ and whose index in $G / P$ is finite. Clearly $V / P$ does not contain $W / P$ either. Since $V / P$ and $W / P$ are normal subgroups of $G / P$, and since $(V / P) \cap(W / P)<W / P$, the minimality of $W / P$ implies $(V / P) \cap(W / P)=1$ or $V \cap W=P$. Hence

$$
W / P=W /(W \cap V) \simeq W V / V \leqq G / V \simeq(G / P) /(V / P)
$$


is finite. By (e.3) therefore $W=P$; and this contradicts our choice of $W / P$ as a minimal normal subgroup of $G / P$.

Case 2. $J(G / P) \neq 1$. Since $Z[J(G / P)]$ is a characteristic and abelian subgroup of $G / P, Z[J(G / P)]=1$ by (e.4). Denote by $K$ the uniquely determined normal subgroup of $G$ which contains $P$ and satisfies $J(G / P)=K / P$. Then $P<K$ and $K$ is the intersection of all those subgroups of finite index in $G$ which contain $P$. It is a consequence of $\left(\mathrm{d}^{\prime}\right)$ and the normality of $K$ that

$$
G / K C(K<G) \text { is finite. }
$$

If $X / P$ is a normal subgroup of $J(G / P)$, then $X$ is a normal subgroup of $K$ so that

$$
K C(K<G) \leqq K C(X<G) \leqq N(X<G) ;
$$

and consequently $[G: N(X<G)]$ is finite. By $\left(\mathrm{d}^{\prime}\right), N(X<G) / X C(X<G)$ is finite. Hence $X C(X<G)$ is a subgroup of finite index in $G$ which contains $P$; and this implies $K \leqq X C(X<G)$. Consequently

$$
J(G / P)=(X / P) C[(X / P)<J(G / P)] ;
$$

and this implies

$$
(X / P) \cap C[(X / P)<J(G / P)] \leqq Z[J(G / P)]=1 .
$$

Thus we have shown the following fact.

(+) If $X$ is a normal subgroup of $J(G / P)$, then $J(G / P)$ is the direct product of $X$ and $C(X<J(G / P))$.

Assume now by way of contradiction the existence of a properly descending sequence of normal subgroups $X_{i}$ of $J(G / P)$. If we let $Y_{i}=C\left(X_{i}<J(G / P)\right)$ then, by $(+)$,

$$
J(G / P)=X_{i} \otimes Y_{i},
$$

and we deduce from $X_{i}<X_{i-1}$ that

$$
X_{i-1}=X_{i} \otimes\left(Y_{i} \cap X_{i-1}\right) \text { and } \quad Y_{i} \cap X_{i-1} \neq 1 .
$$

Since $G$ is a torsion group, every $Y_{i} \cap X_{i-1}$ contains an element $y_{i}$ of order a prime; and it is fairly clear that these elements $y_{i}$ generate an infinite abelian subgroup of $G / P$ all of whose elements are of squarefree order; and this is impossible by (e.4). This contradiction shows that the minimum condition is satisfied by the normal subgroups of $J(G / P)$.

Since $J(G / P) \neq 1$, there exists a minimal normal subgroup $L$ of $J(G / P)$. By $(+), J(G / P)=L \otimes C(L<J(G / P))$. It follows that every normal subgroup of $L$ is a normal subgroup of $J(G / P)$. By the minimality of $L$ we find now that $L$ is simple. Application of condition (4.b) shows that $L$ is finite. We recall that $J(G / P)=K / P$ and that clearly $K C(K<G) / P \leqq N(L<G / P)$. But 
$G / K C(K<G)$ is finite, by $\left(\mathrm{d}^{\prime}\right)$. The normalizer of $L$ in $G / P$ has therefore finite index in $G / P$; and this shows that the class of conjugate subgroups of $L$ is finite. Since every subgroup conjugate to $L$ in $G / P$ is a finite normal subgroup of $J(G / P)$, it follows now that $L$ is contained in a finite normal subgroup of $G / P$ [which is part of $J(G / P)$ ]. Application of (e.3) shows that $L=1$, a contradiction.

Thus we have been led to a contradiction in either case by assuming that $P<G$. Hence $G=P$. By (e.2), $M$ is an abelian subgroup of finite index in $P=G$; and by $\left(\mathrm{a}^{\prime}\right)$ the minimum condition is satisfied by the abelian subgroups of $G$. It is clear now that the minimum condition is satisfied by the subgroups of $G$; and we have derived condition (1) from (4). This completes the proof.

12. It is customary to term $p$-group, for $p$ a prime, any group all of whose elements are of order a power of $p$. Every group contains a maximal $p$-subgroup; and these take, in the general theory, the place of the Sylow subgroups of finite groups. It is known that, in general, maximal $p$-subgroups need not be conjugate; see, for instance, Baer [1]. Thus the following result may be of some interest.

CoROllary. If the minimum condition is satisfied by the subgroups of the group $G$, and if there exists an abelian subgroup of finite index in $G$, then any two maximal p-subgroups of $G$ are conjugate in $G$.

Proof. By our hypothesis there exists an abelian normal subgroup $N$ of finite index in $G$. We denote by $P$ the totality of elements of order a power of $p$ in $N$; and we denote by $Q$ the totality of elements of order prime to $p$ in $N$. Clearly $P$ and $Q$ are characteristic subgroups of the abelian group $N$, and $N$ is their direct product: $N=P \otimes Q$. Since $N$ is a normal subgroup of $G, P$ and $Q$ are normal subgroups of $G$.

If $R$ is a finitely generated subgroup of $G$, then $R /(R \cap N) \simeq N R / N$ is finite as a subgroup of the finite group $G / N$. Since $R$ is finitely generated and $R /(R \cap N)$ is finite, $R \cap N$ is finitely generated; see Baer [5; $\S 1$, Finiteness Principle]. But finitely generated subgroups of abelian torsion groups are finite. Hence $R \cap N$ is finite so that $R$ itself is finite.

Consider now some maximal $p$-subgroup $S$ of $G$. Since $P$ is a normal $p$-subgroup of $G, P S$ is a $p$-group. By the maximality of $S$ we have therefore $P S=S$ or $P \leqq S$. Since $Q \cap S=1$, it follows that $P=N \cap S$. Consequently $S / P=S /(N \cap S) \simeq N S / N$ is finite as a subgroup of the finite group $G / N$.

Consider now two maximal $p$-subgroups $H$ and $K$ of $G$. Then $N \cap H=P$ $=K \cap N$ and both $H / P$ and $K / P$ are finite. Consequently there exists a finitely generated subgroup $H^{*}$ of $H$ such that $H=P H^{*}$; and there exists a finitely generated subgroup $K^{*}$ of $K$ such that $K=P K^{*}$. Then $R=\left\{H^{*}, K^{*}\right\}$ is a finitely generated subgroup of $G$; and we have shown before that every 
finitely generated subgroup $R$ of $G$ is finite. It is clear that $H^{*} \leqq R \cap H$. Since $H$ is a $p$-group, $R \cap H$ is a $p$-subgroup of the finite group $R$; and as such $R \cap H$ is part of a $p$-Sylow subgroup $U$ of $R$. We note that

$$
H=P H^{*} \leqq P(R \cap H) \leqq P U .
$$

Since $P$ is a normal $p$-subgroup of $G$ and since $U$ is a finite $p$-group, $P U$ is a $p$-subgroup of $G$. By the maximality of $H$ we find that $H=P U$. Hence $R \cap H \leqq U \leqq R \cap H$ or $R \cap H=U$ is a $p$-Sylow subgroup of $R$. Likewise we see that $R \cap K$ is a $p$-Sylow subgroup of $R$ and that $K=P(R \cap K)$. But any two $p$-Sylow subgroups of the finite group $R$ are conjugate in $R$. Consequently there exists an element $r$ in $R$ such that $r^{-1}(R \cap H) r=R \cap K$; and this implies

$$
r^{-1} H r=r^{-1} P(R \cap H) r=P(R \cap K)=K .
$$

This completes the proof.

REMARK. It is well known that any two maximal $p$-subgroups of some group $G$ are conjugate in $G$, if there exists only a finite number of maximal $p$-subgroups of $G$; see, for instance, Baer [1]. But this theorem cannot be anplied in our case, since groups of the type considered by us may easily contain an infinity of maximal $p$-subgroups, as may be seen from easily constructed examples; see, for instance, $\S 7$, Remark 6.

\section{BIBLIOGRAPHY}

REINHOLd BAER

1. Sylow theorems for infinite groups, Duke Math. J. vol. 6 (1940) pp. 598-614.

2. Finiteness properties of groups, Duke Math. J. vol. 15 (1948) pp. 1021-1032.

3. Groups with descending chain condition for normal subgroups, Duke Math. J. vol. 16 (1949) pp. 1-22.

4. Endlichkeitskriterien für Kommutatorgruppen, Math. Ann. vol. 124 (1952) pp. 161-177.

5. The hypercenter of a group, Acta Math. vol. 89 (1953) pp. 165-208.

B. H. NeumanN

1. Groups with finite classes of conjugate elements, Proc. London Math. Soc (3) vol. 1 (1951) pp. 178-187.

UNIVERSITY OF ILLINOIS, URBANA, Ill. 